\title{
Histomorphological Spectrum of Renal Lesion in An Autopsy Study
}

\author{
Vaneet Kaur Sandhu*, Arun Puri and Navtej Singh \\ Department of Pathology, Guru Gobind Singh Medical College and Hospital ,Faridkot , Punjab,INDIA
}

\begin{abstract}
Background: Autopsy provides normal as well as diseased human tissue for morphologic studies, for establishment of cell and organ culture as well for xenotransplantation. It provides the opportunity to discover new diseases, to evaluate toxic effects of drugs and therapies. The kidneys are often affected by chronic inflammatory lesions, neoplasms, toxic effects of various drugs and metabolic disorders.

Material and Methods: This was a five year study from January 2011 to December 2015 in our department of pathology. The kidneys of medico legal autopsies performed during these years were subjected to our study. After excluding 30 cases of severely damaged tissue, 120 cases of well preserved renal medico legal autopsies were included in our study. The stained microscopic sections were examined by two histopathologists independently.

Results: Seventy five of the 120 autopsies were males, while 45 were females. In $27(22.5 \%)$ cases, the microscopic morphology was close to normal histology. Remaining 93(77.5\%) cases had a nephropathological findings The percentage of non glomerular nephropathies (60.8\%) was higher as compared to that of glomerular lesions (16\%). $20(16 \%)$ cases exhibited glomerular alterations such focal global glomerular sclerosis, segmental glomerular sclerosis, nodular mesangial sclerosis, basement thickening and mesangial cellular proliferation. Tubular and interestium lesions were observed in $34.16 \%$ which included acute tubular necrosis, chronic pyelonephritis and tubercular pyelonephritis. Renal arteriosclerosis was observed in $25 \%$ cases. Renal cell carcinoma was incidentally detected in $1.6 \%$
\end{abstract}

Conclusion: Our study provided satisfactory data in respect to morphological spectrum of various renal lesions in autopsy.

Keywords: Autopsy, Renal diseases,

\section{Introduction}

The autopsy data continue to embellish the medical literature; it provides a unique opportunity for physicians to correlate their physical and laboratory findings with the pathologic changes of disease process. The autopsy pathologists uncover the changing patterns of disease. The autopsy aids in the education of students in medicine and other health related disciplines by providing teaching material for anatomy, histology and pathology. Further it plays an important role in establishment of diagnosis and whenever possible determines the possible cause of the death.

Renal diseases are responsible for great deal of morbidity. Chronic kidney disease is now recognized as a major global public health problem and is an independent risk factor for cardiovascular disease ${ }^{[1-2]} \mathrm{CKD}$ affects $10-15 \%$ of the adult population worldwide. ${ }^{[3-4]}$ The increased prevalence of kidney diseases is a consequence of the accumulation of risk factors such as hypertension, diabetes, dyslipidaemia and obesity. ${ }^{[5]}$ Pathologic examination of renal tissue in autopsy throws a light on renal histologic changes in the general population, might provide useful information for preventing chronic renal diseases that tend to be asymptomatic and often go undiagnosed.

The aim of the present study was to analyze varied spectrum of renal lesions detected on autopsy.

\section{Material and Methods}

This was a five year study from January 2011 to December 2015 in our department of pathology. The kidneys of medico legal autopsies performed during these years were subjected to our study. After excluding 30 cases of severely damaged tissue, 120 cases of well preserved renal medico legal autopsies were included in our study. The data pertaining to age, gender, and clinical findings were recorded from deceased post mortem papers. The thorough gross examination including weight, measurements, colours were recorded and then tissue was fixed in 10\% neutral buffered formalin. The formalin fixed tissues were sampled, each sample included the cortico-medullary region then were further processed by automatic processor. The three micrometer thick sections were obtained from paraffin embedded tissue samples and were histochemically stained with haematoxylin and eosin. The special stains periodic acid Schiff reagent and silver methanamine were 
done as and when required. The microscopic sections were observed by two histopathologists indepentantly. The stenosis of renal arteries were graded on the basis of luminal narrowing of arteries and was grade from grade 0 ( normal ) to grade IV( complete obstruction)

Grade 0: normal, Grade I: 1-25\% narrowing of lumen, Grade II: $26-50 \%$ narrowing of lumen, Grade III: $51-75 \%$ narrowing of lumen, Grade IV: 76-100 narrowing of lumen.

\section{Results}

The age range of the autopsies was between 25 and 80 years. Seventy five of the 120 autopsies were males, while 45 were females. In $27(22.5 \%)$ cases, the microscopic morphology was close to normal histology. Remaining 93(77.5\%) cases had a nephropathological finding at autopsy. Table 1 summarizes the various renal lesions in renal autopsies. The percentage of non glomerular nephropathies $(60.8 \%)$ was higher as compared to that of glomerular lesions (16\%).

Glomerular Findings: In 20 cases of renal autopsies glomerular alterations were observed of which 12 males and remaining 8 were were females. Ten cases $(8.3 \%)$ exhibited focal global sclerosis. There were two cases $(1.6 \%)$ of segmental glomerular sclerosis and three $(2.5 \%)$ cases exhibited nodular glomerular sclerosis. Basement membrane thickening was observed in two $(1.6 \%)$ cases. Three $(2.5 \%)$ cases showed moderate degree of mesangial cellular proliferation.
Tubular and Interestium Findings:. There were tubular and interestium alterations in 41(34.16\%) cases of which 29 were males and twelve were females. Acute tubular necrosis was seen in $27(22.5 \%)$ cases of renal autopsies fig1. The chronic pyelonephritis fig2 was noted in $8(6.6 \%)$ cases out which 5 cases had diffuse interstitial fibrosis, tubular atrophy and diffuse global sclerosis. Remaining three cases had focal interstitial inflammation with focal tubular atrophy fig 3 . In addition to chronic pyelonephritis three were accompanied by hydronephrosis and two cases had nephrocalcinosis. The tubercular pyelonephritis was observed in 6 (5\% cases) of renal autopsies. The caseating epitheloid cell granulomas, langhan type of giant cells were noted in cases of tuberbular pyelonephritis fig 4 and fig 5. The Ziehl Neelson stain exhibited acid fast bacilli.

Vascular Findings: The most frequent histological diagnosis was renal arteriolosclerosis observed in 30 $(25 \%)$ of renal autopsies, of which 20 were males and 10 were females. Grade II renal arteriolosclerosis was observed in $18(60 \%)$ cases followed by Grade I renal arteriolosclerosis in $6(20 \%)$, Grade III in $4(13.3 \%)$ and remaining had grade IV renal arteriolosclerosis $2(6.6 \%)$. The arterial nephrosclerosis was characterized by intimal fibrous thickening of arteries fig [6]

The incidental renal masses in the study comprised of clear cell renal cell carcinoma in two renal autopsies, of which one was 65 year old male and other being 55 year old female.

Table 1: Distribution of various renal findings on autopsy study (120).

\begin{tabular}{|l|c|c|}
\hline Histopathological findings & Number & Gender M F \\
\hline A.Glomerular lesions & $20(16.6 \%)$ & 128 \\
1.Focal global glomerular sclerosis & $10(8.3 \%)$ & 73 \\
2.Segmental glomerular sclerosis & $2(1.6 \%)$ & 11 \\
3.Nodular glomerular sclerosis & $3(2.5 \%)$ & 21 \\
4.Mesengium proliferation & $3(2.5 \%)$ & 12 \\
5.Basement membrane thickening & $2(1.6 \%)$ & 11 \\
\hline B. Tubular and Interstitial findings & $41(34.16 \%)$ & 2912 \\
1.Acute tubular necrosis & $27(22.5 \%)$ & 207 \\
2.Chronic pyleonephritis & $8(6.6 \%)$ & 53 \\
3.Tubercular pyleonephritis & $6(5 \%)$ & 42 \\
\hline C. Vascular findings & $30(25 \%)$ & 2010 \\
1. Renal arteriosclerosis & $18(60 \%)$ & 153 \\
a.Grade II & $6(20 \%)$ & 24 \\
b.Grade III & $4(13.3 \%)$ & 22 \\
c.Grade I & $2(6.6 \%)$ & 11 \\
d.Grade IV & & \\
\hline D.Neoplasm & & 11 \\
1.Renal cell carcinoma & $2(1.6 \%)$ & 1314 \\
\hline E.Normal histology & $27(22.5 \%)$ & $\mathbf{7 5 4 5}$ \\
\hline Total & $\mathbf{1 2 0}$ & \\
\hline
\end{tabular}




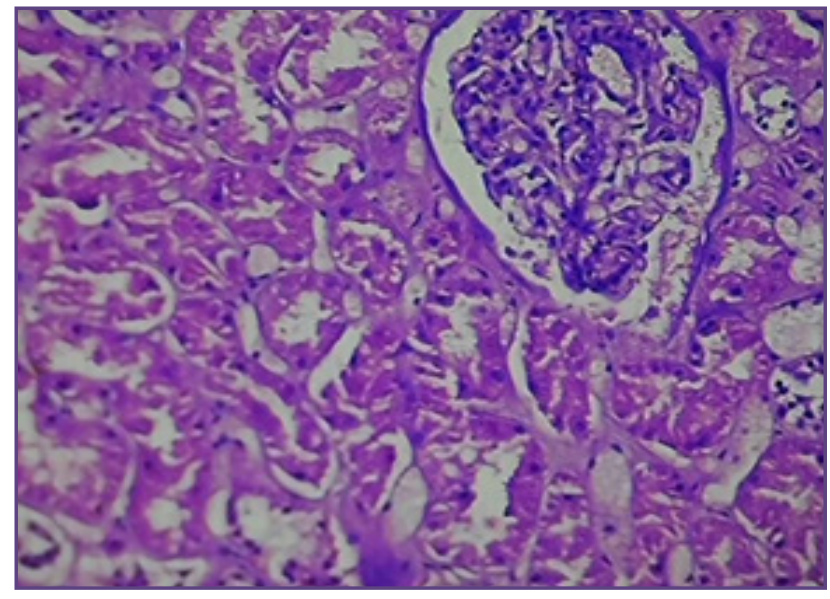

Fig. 1: Sections shows a viable glomerulus with tubular epithelial cells exhibiting necrosis in case of Acute tubular necrosis H\& E x 40.

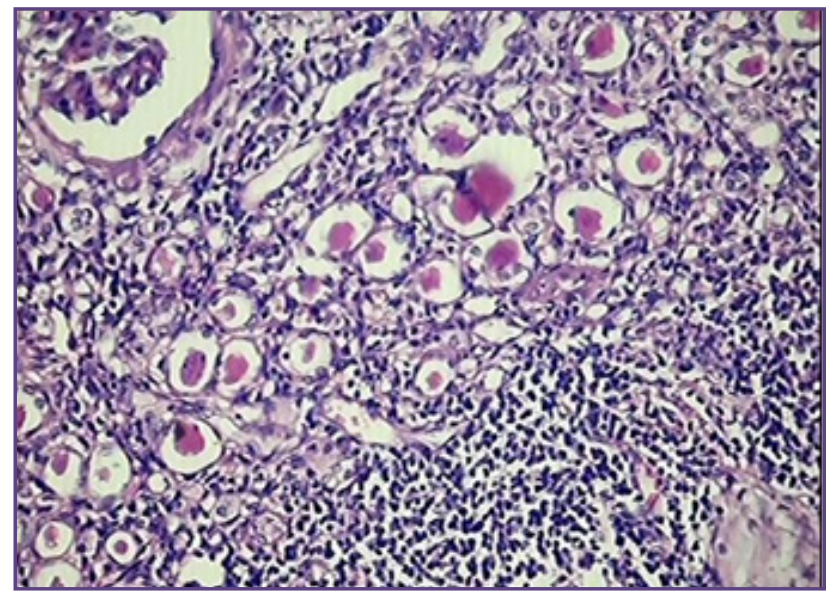

Fig. 3: Sections shows dense inflammatory infiltrate in the interestium with thyroidization of tubules in case of chronic pyleonephritis H\& E x 40.

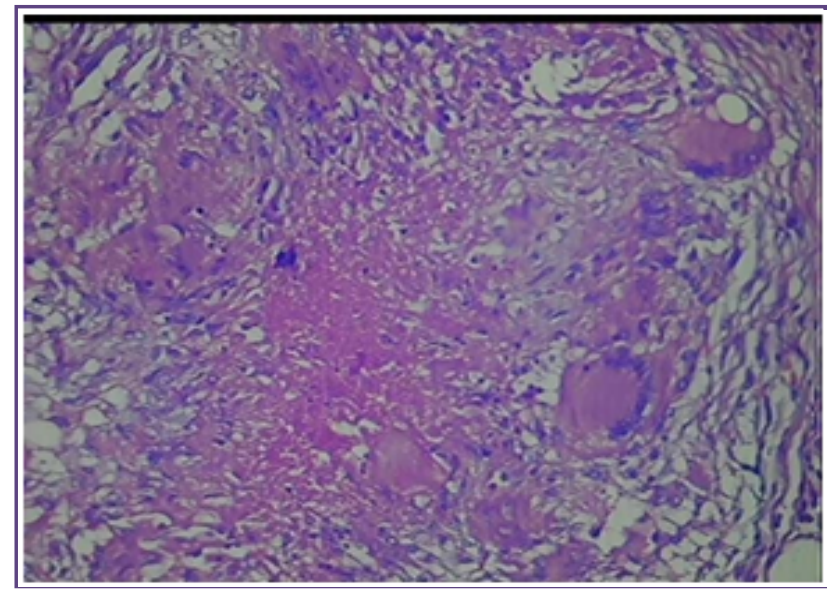

Fig. 5: High power view of caseating epitheloid cell granulomas in case of tubercular pyleonephritis H \& E x 40

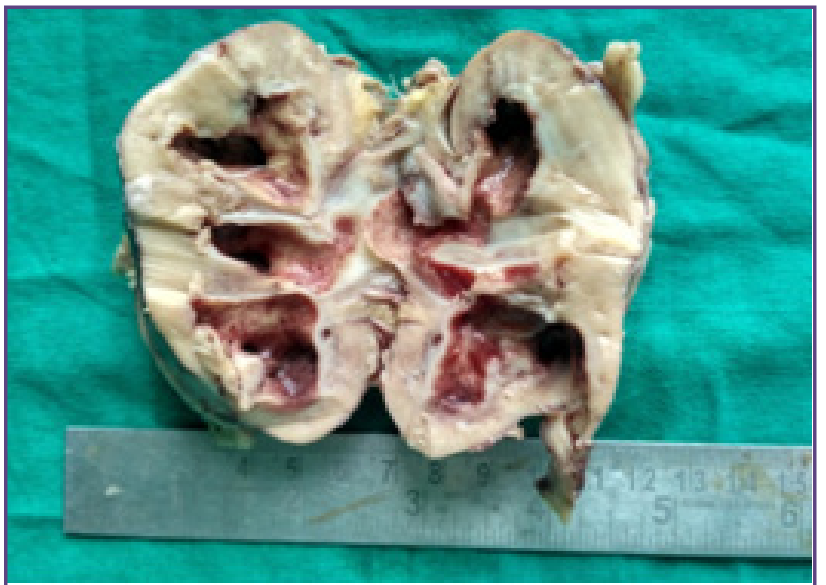

Fig. 2: Gross appearance of a kidney from a case of chronic pyleonephritis shows dilation and blunting of pelvic calyces.

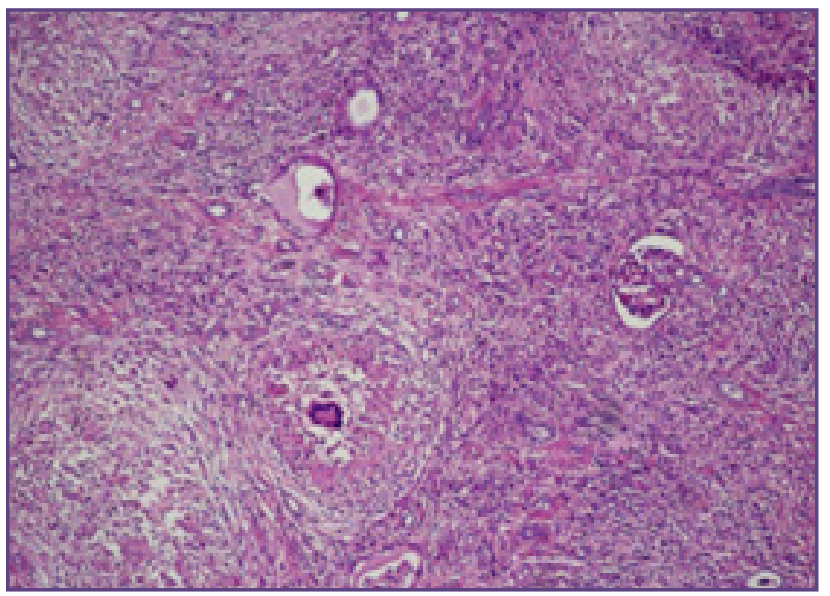

Fig. 4: Sections show epitheliod cell granulomas in the interestium in case of tuberbular pyleonephritis $\mathrm{H} \& \mathrm{E} \times 10$

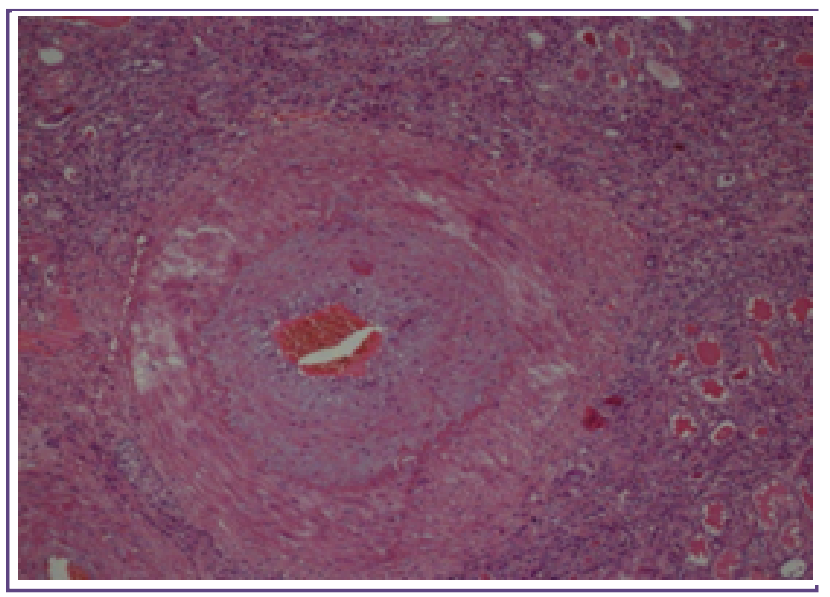

Fig . 6: Sections show renal artery exhibiting arteriosclerosis with thickening of the wall and narrowing of the lumen H\& E 10 


\section{Discussion}

The distribution of renal lesions vary with geographic area, age, gender, environmental, nutritional and genetic factors. ${ }^{[-7]}$ In current analysis in 27 cases the microscopic findings were close to normal histology. This is in concordance with study conducted by Usta et al ${ }^{[8]}$ on 55 renal autopsy in which 23 cases exhibited almost normal histology. We observed nephropathological changes in $77.5 \%$ of renal autopsies .How ever slightly lower percentage of renal lesions were obtained by Monga et al. ${ }^{[9]}$ and Martinez et al. ${ }^{[10]}$ in their respective work on renal autopsies who found renal lesions in $68 \%$ and $59 \%$ cases respectively. The histopathologic findings in the present study revealed presence of non glomerular nephropathies in $73(60.8 \%)$ cases and glomerular lesions $20(16 \%)$ cases .A study conducted by Hailemariam S et al. ${ }^{[11]}$ on 237 autopsies observed presence of glomerular or vascular pathology in 28\% ,non glomerular lesion in 33\% and 29\% had combined lesions.

We observed glomerular sclerosis in $20(16.6 \%)$ cases of which $10(8.3 \%)$ cases exhibited global focal glomerular sclerosis, two $(1.6 \%)$ cases had segmental glomerular sclerosis, three $(2.5 \%)$ cases had nodular sclerosis, three $(2.5 \%)$ cases had moderate to severe degree of mesangial cellular proliferation and two (1.6\%) case had basement membrane thickening. However Usta et al ${ }^{[8]}$ in their work observed focal global sclerosis in eleven cases, followed by 12 cases of mesangial cellular proliferation and one case of basement membrane thickening.

In current analysis $30(25 \%)$ cases exhibited renal arteriosclerosis .Mc Namara BJ et al. ${ }^{[12]}$ in their work on 81 renal autopsies reported arteriolar nephrosclerosis in $34 \%$ cases . Nephrosclerosis at autopsy is associated with increasing age and is more frequent in blacks than whites. ${ }^{[13]}$ Some observers suggest that renal arteriosclerosis is strongly linked with hypertension. ${ }^{[14-16]}$

Among present work tubular and interstitium changes were observed in 41 (34.16\%) cases of which27 (22.5\%) cases had acute tubular necrosis. This might be attributed to death due to intake of toxic substance, drugs over dose and snake bite. Renal tuberculosis and chronic pyelonephritis were observed in $6(5 \%)$ and $8(6.6 \%)$ of cases respectively in our work. Tuberculosis has been described as a global emergency by WHO and in developing countries it still remains as a major cause of morbidity and mortality. ${ }^{[17]}$ Renal tuberculosis develops in approximately $5 \%$ of patients with active tuberculosis. Renal tuberculosis usually remains clinically silent and often detected incidentally in autopsy studies. ${ }^{[18]}$
Two (1.6\%) cases of renal cell carcinoma (clear cell type) were observed during our study. Kozlowska Jolanta et al. ${ }^{[19]}$ in their work observed renal tumors in $2.76 \%$ cases in post mortem examination. Shah VB et al. ${ }^{[20]}$ and Sapna $\mathrm{P}$ et al. ${ }^{[21]}$ in their respective autopsy studies revealed 5 cases and 4 cases of renal masses detected incidentally.

Conclusion There is still no substitute for autopsy study which throws immense light on pathogenesis of disease, reveals hazardous effects of therapies and drugs administered and lastly often reveal cause of death. The present study on renal autopsies showed renal vascular and tubulointerstitial lesions outnumbered in comparison to glomerular lesions. We observed $1.5 \%$ cases of renal cell carcinoma. Our study provided satisfactory data in respect to morphological spectrum of various renal lesions in an autopsy study however it does not reflect the actual incidence of renal lesions in a population.

\section{Referances}

1. Levey AS, Atkins R, Coresh J. Cohen EP, Collins AJ, Ec Kardt KU. Chronic kidney disease as a global public health problem:approaches and initiatives- a position statement from Kidney Disease Improving Global Outcomes. Kidney Int 2007;72:247-259.

2. Schiffrin EL, Lipman ML, Mamm JF. Chronic kidney disease:effects on the cardiovascular system. Circulation 2007;116:85-97.

3. Imai E, Horio M, Watanabe T, Iseki K, Yamagata K, Hara S. Prevalence of chronic kidney disease in the Japanese general population.Clin Exp Nephrol 2009;13:621-630.

4. Hall YN, Hsu CY, Iribarren C Darbiman J, Mc Culloch CE, Go Alan S. The conundrum of increased burden of end stage renal disease in Asians. Kidney Int 2005;68:2310-2316.

5. Nagata M, Ninomiya T, Doi Y, Yonemolo K, Kubo M, Hata J. Trends in the prevalence of chronic kidney disease and its risk factors in a general population:the Hisayama Study. Nephrol Dial Transplant 2010;25:2557-2564.

6. Kurnatowaska I, Jedrzejka D Malyska A, Danilewicz MW, Danilewicz M, Nowicki M. Trends in the incidence of biopsy proven glomerular dieases in the adult population in central Poland in the years1990-2010.Kidney Blood Press Res 2012;35:254-258.

7. Zaza G, Bernich P, Lupo A. Triveneto Register of Renal Biopsies (TVRRB).Incidence of primary glomerulonephritis in a large North Eastern Italian area:a 13 year renal biopsy study.Nephrol Dial Transplant 2013;28:367-372.

8. Usta U, Tastekin E, Isler E, Kutlu AK, Puyan FO. Histopathological and immune alterations in autopsied kidneys.Saudi Med J 2014;35:1331-38.

9. Monga G, Mazzucco G, Boldorini R, Cristina S, Giacalone A, Fortunato $\mathrm{M}$ et al. Renal changes in patients with acquired immunodeficiency syndrome.a postmortem study on an 
unselected population in northwestern Italy.Mod Pathol 1997;10(3):159-67.

10. Martinez PM, Penta JM, Arnalich F, Garcia M, Adridanto $\mathrm{O}$, Gonzalez J et al. Spectrum of renal pathology in HIV infection:descriptive of 85 autopsies and clinic-pathologic correlation.Rev ClinEsp 1996;196(9):557-83.

11. Hailemariam S, Walder M, Burger HR, et al.Renal pathology and premortem clinical presentation of Caucasian patients with AIDS: An autopsy study from the era prior to antiretroviral therapy.Swiss Med Wkly2001;131:412-17.

12. Bridgette J. Mc Namara, Boucar Diouf, et al. Renal pathology, glomerular number and volume in a West African urban community. Nephrol Dial Transplant2008;23:2576-2585.

13. Marcantoni C, Fogo AB:A perspective on arterionephrosclerosis: from pathology to pathogenesis.J Nephrol .2007;20:518.

14. Tracy RE. Recent declines of hypertensiverenovasculopathies in New Orelans blacks.Am J Hypertens 2000;13:966-972.
15. Tracy RE, Rios-Dalenz JL. Rarity of hypertensive stigmata in aging renocortical arteries of Bolivians. Virchows Archiv 1994:424:307-314.

16. Tracy RE, Mulvad G, Pederson HS. Blood pressure in people in Greenland assed by renovasculopathies of hypertension at autopsy. Am J Hypertens 1996;9:560-565.

17. Rastogi P, Kanchan T, Menezes RG. Sudden unexpected deaths due to tuberculosis.An autopsy based study.Journal of Forsensic Medicine and Toxicology.2011;28:81-5.

18. Eastwood JB, Corbishley CM, Grange JM. Tuberculosis and the kidney.J AMSoc Nephrol.2000;12:1307-1314.

19. Kozlowska J, Okon K. Renal tumors in postmortem material. Pol J Pathol.2008;59:21-25.

20. Shah VB, Deokar MS. Spectrum of incidental renal masses detected at autopsy.Bombay Hosp J.2009;51:432-36

21. Patel S, Rajalakshmi BR, Manjunath GV. Histopathologic findings in autopsies with emphasis on interesting and incidental findings-A Pathologists Perspective.J of Clinicla and Diagnostic Research2016;10:8-12.

*Corresponding author:

Dr Vaneet Kaur Sandhu, Associate Professor, Department of Pathology, Guru Gobind Singh Medical College and Hospital Faridkot 151203 Punjab India Phone: +91 9815683434

Email: vaneetsandhu@gmail.com 